ANNALES

POLONICI MATHEMATICI

$83.2(2004)$

\title{
Global solutions for a functional second order abstract Cauchy problem with nonlocal conditions
}

\author{
by Eduardo Hernández M. (São Paulo) and \\ Hernán R. Henríquez (Santiago)
}

\begin{abstract}
By using the theory of strongly continuous cosine families and the properties of completely continuous maps, we study the existence of mild, strong, classical and asymptotically almost periodic solutions for a functional second order abstract Cauchy problem with nonlocal conditions.
\end{abstract}

1. Introduction. The purpose of this work is to study the existence of mild, strong and classical solutions of a class of functional second order differential equations of the form

$$
\begin{aligned}
& x^{\prime \prime}(t)=A x(t)+f\left(t, x(t), x(a(t)), x^{\prime}(t), x^{\prime}(b(t))\right), \quad t \in I, \\
& x(0)+p\left(x, x^{\prime}\right)=x_{0}, \\
& x^{\prime}(0)+q\left(x, x^{\prime}\right)=x_{1},
\end{aligned}
$$

where $A$ is the infinitesimal generator of a strongly cosine family $(C(t))_{t \in \mathbb{R}}$ of bounded linear operators on a Banach space $X ; I$ denotes a finite interval $[0, T]$ or the interval $[0, \infty)$ and $a(\cdot), b(\cdot): I \rightarrow I$; and $p(\cdot), q(\cdot): C(I ; X) \times$ $C(I ; X) \rightarrow X$ and $f: I \times X^{4} \rightarrow X$ are appropriate functions.

In general, the nonlocal conditions can be applied in physics with better effect than the classical initial conditions $x(0)=x_{0}$. For the importance of nonlocal conditions in different fields, we refer to [1-5] and the references therein.

The study of nonlocal initial value problems was initiated by Byszewski in $[1,5]$. Specifically in [1] Byszewski proves the existence of mild, strong

2000 Mathematics Subject Classification: 47D09, 34G10.

Key words and phrases: abstract Cauchy problem, cosine functions of operators, almost periodic functions.

This work was supported by DICYT-USACH, Project 04-0133HM, and FONDECYT, Project 1020259. 
and classical solutions of the semilinear nonlocal Cauchy problem

$$
\begin{aligned}
& x^{\prime}(t)=A x(t)+f(t, x(t)), \quad t \in I=[0, T], \\
& x(0)=x_{0}+q\left(t_{1}, \ldots, t_{n}, x(\cdot)\right) \in X,
\end{aligned}
$$

where $A$ is the infinitesimal generator of a strongly continuous semigroup of linear operators on $X ; 0<t_{0}<t_{1}<\cdots<t_{n} \leq T$; $f:[0, T] \times X \rightarrow X$ and $q(\cdot, \cdot): I^{n} \times C(I ; X) \rightarrow X$ are appropriate functions, and the expression $q\left(t_{1}, \ldots, t_{n}, x(\cdot)\right)$ only involves the values of $x$ at some of the points $t_{i}$, for instance $q\left(t_{1}, \ldots, t_{n}, x(\cdot)\right)=\sum_{i=1}^{n} \alpha_{i} x\left(t_{i}\right)$.

Some second order nonlocal initial value problems have been studied by Ntouyas \& Tsamatos in $[14,15]$. They discuss the existence of solutions for a second order delay integrodifferential equation with nonlocal conditions of the form

$$
\begin{aligned}
x^{\prime \prime}(t) & =A x(t)+f\left(t, x\left(\sigma_{1}(t)\right), \int_{0}^{t} k(t-s) h\left(s, x\left(\sigma_{2}(s)\right), x\left(\sigma_{3}^{\prime}(s)\right)\right) d s, x\left(\sigma_{4}(t)\right)\right), \\
x(0) & =g(x)+x_{0}, \quad x^{\prime}(0)=\eta,
\end{aligned}
$$

where $A$ is the generator of a strongly continuous cosine family, $(C(t))_{t \in \mathbb{R}}$, of bounded linear operators on $X, x_{0}, \eta \in X$ and $g: C(I ; X) \rightarrow X, f, h$ : $I \times X^{3} \rightarrow X$ are appropriate functions. In general the results are obtained under the strong assumption that $C(t)$ is compact for every $t>0$, which in turn implies that $\operatorname{dim}(X)<\infty$.

Throughout this paper $X$ will be a Banach space endowed with a norm $\|\cdot\|$ and $C(t)$ denotes a strongly continuous cosine operator function defined on $X$ with infinitesimal generator $A$. We refer the reader to $[9,16]$ for the necessary background about cosine functions. We only mention a few results and notations needed to establish our results. We denote by $S(t)$ the sine function associated with $C(t)$ which is defined by

$$
S(t) x:=\int_{0}^{t} C(s) x d s, \quad x \in X, t \in \mathbb{R} .
$$

For a closed operator $B: D(B) \subset X \rightarrow X$ we denote by $[D(B)]$ the space $D(B)$ endowed with the graph norm $\|\cdot\|_{B}$. In particular $[D(A)]$ is the space $D(A)$ endowed with the norm

$$
\|x\|_{A}=\|x\|+\|A x\|, \quad x \in D(A) .
$$

The notation $E$ stands for the space of all vectors $x \in X$ for which the function $C(\cdot) x$ is of class $C^{1}$. It was proved by Kisyński [12] that $E$ endowed with the norm

$$
\|x\|_{1}=\|x\|+\sup _{0 \leq t \leq 1}\|A S(t) x\|, \quad x \in E
$$


is a Banach space. The operator-valued function

$$
G(t)=\left[\begin{array}{ll}
C(t) & S(t) \\
A S(t) & C(t)
\end{array}\right]
$$

is a strongly continuous group of linear operators on the space $E \times X$ generated by the operator $\mathcal{A}=\left[\begin{array}{ll}0 & I \\ A & 0\end{array}\right]$ defined on $D(A) \times E$. It follows that $A S(t): E \rightarrow X$ is a bounded linear operator and $A S(t) x \rightarrow 0$ as $t \rightarrow 0$, for each $x \in E$. Furthermore, if $x:[0, \infty) \rightarrow X$ is a locally integrable function then

$$
y(t)=\int_{0}^{t} S(t-s) x(s) d s
$$

defines an $E$-valued continuous function. This is an immediate consequence of the fact that

$$
\int_{0}^{t} G(t-s)\left[\begin{array}{c}
0 \\
x(s)
\end{array}\right] d s=\left[\begin{array}{l}
t \\
\int \\
0 \\
t \\
\int_{0}^{t} C(t-s) x(s) d s
\end{array}\right]
$$

defines an $E \times X$-valued continuous function.

The existence of solutions for the second order abstract Cauchy problem

$$
\begin{aligned}
x^{\prime \prime}(t) & =A x(t)+h(t), \quad 0 \leq t \leq T, \\
x(0) & =x_{0}, \quad x^{\prime}(0)=x_{1},
\end{aligned}
$$

where $h:[0, T] \rightarrow X$ is an integrable function, has been discussed in [17]. Similarly, the existence of solutions of the semilinear second order abstract Cauchy problem has been treated in [18]. We only mention here that the function $x(\cdot)$ given by

$$
x(t)=C(t) x_{0}+S(t) x_{1}+\int_{0}^{t} S(t-s) h(s) d s, \quad 0 \leq t \leq T,
$$

is called a mild solution of (1.6)-(1.7). If $x_{0} \in E$ then $x(\cdot)$ is continuously differentiable and

$$
x^{\prime}(t)=A S(t) x_{0}+C(t) x_{1}+\int_{0}^{t} C(t-s) h(s) d s .
$$

Further terminology and notations are those generally used in functional analysis. In particular, $\mathcal{L}(Z ; Y)$ stands for the Banach space of bounded linear operators from a Banach space $Z$ into a Banach space $Y$, and $B_{r}(x)$ for the closed ball with center at $x$ and radius $r$ in an appropriate space. Moreover, for bounded functions $\xi: I \rightarrow \mathbb{R}$ and $\Lambda: Y \rightarrow X$ we set $\xi_{t}=$ $\sup \{\xi(\theta): \theta \leq t\}$ and $N_{\Lambda}=\sup \{\|\Lambda(y)\|: y \in Y\}$. 
The paper is organized as follows. In Section 2 we establish the existence of mild, strong and classical solutions of some second order nonlocal initial value problems in a finite interval, and in Section 3 we consider the existence of $g$-bounded solutions and asymptotically almost periodic solutions on $[0, \infty)$. Finally, in Section 4 we present an application to the wave equation. The results are obtained using the techniques introduced in Henríquez [11], the ideas in Ntouyas \& Tsamatos [14, 15] and the following result on existence of fixed points due to Schaefer (see [7, Corollary 8.1]).

Lemma 1.1. Let $D$ be a convex subset of a Banach space $X$ and assume that $0 \in D$. Let $F: D \rightarrow D$ be a completely continuous map. Then either the map $F$ has a fixed point in $D$ or the set $\{x \in D: x=\lambda F(x), 0<\lambda<1\}$ is unbounded.

2. Existence of solutions. In this section we study the existence of solutions of some nonlocal second order functional abstract Cauchy problem in the interval $I=[0, T]$. We begin by introducing some notations. We assume that $M \geq 1$ and $N \geq 1$ are constants such that $\|C(t)\| \leq M$, $\|S(t)\| \leq N$ for every $t \in I$. Moreover, we consider the space of continuous functions $C(I ; X)$ endowed with the norm $\|\cdot\|_{\infty}$ of uniform convergence, and we abbreviate $C(I ; I)$ to $C(I)$.

In this section we always assume that the following general assumptions hold:

Assumption $\mathrm{A}_{1}$.

(a) $a: I \rightarrow I$ is continuous and $a(t) \leq t$ for every $t \in I$.

(b) $f: I \times X \times X \rightarrow X$ satisfies the following Carathéodory conditions:

(i) $f(t, \cdot): X \times X \rightarrow X$ is continuous for a.e. $t \in I$;

(ii) $f(\cdot, x, y): I \rightarrow X$ is strongly measurable for each $x, y \in X$.

(c) There exists a continuous function $m: I \rightarrow[0, \infty)$ and a continuous nondecreasing function $W:[0, \infty) \rightarrow(0, \infty)$ such that

$$
\|f(t, x, y)\| \leq m(t) W(\|x\|+\|y\|), \quad t \in I, x, y \in X .
$$

(d) $p, q: C(I ; X) \rightarrow X$ are continuous.

First we consider the following second order initial value problem:

$$
\begin{aligned}
& x^{\prime \prime}(t)=A x(t)+f(t, x(t), x(a(t))), \quad t \in I, \\
& x(0)+p(x)=x_{0}, \\
& x^{\prime}(0)+q(x)=x_{1},
\end{aligned}
$$

where $x_{0}, x_{1} \in X$.

Expression (1.8) motivates the following definition. 
Definition 2.1. We say that a function $x(\cdot): I \rightarrow X$ is a mild solution of the problem $(2.1)-(2.3)$ if $x(\cdot)$ is continuous and satisfies the integral equation

$$
\begin{aligned}
x(t)= & C(t)\left(x_{0}-p(x)\right)+S(t)\left(x_{1}-q(x)\right) \\
& +\int_{0}^{t} S(t-s) f(s, x(s), x(a(s))) d s, \quad t \in I .
\end{aligned}
$$

TheOREm 2.1. Assume that the following conditions hold:

(H-1) $\quad p(\cdot)$ and $q(\cdot)$ bounded and both $p(\cdot): C(I ; X) \rightarrow C(I ; X)$ and $x \mapsto$ $S(\cdot) q(x)$ are completely continuous.

(H-2) For each $t \in I, t^{\prime} \leq t$ and every constant $L \geq 0$ the set $\left\{S\left(t^{\prime}\right) f(s, x, y)\right.$ : $0 \leq s \leq t,\|x\|,\|y\| \leq L\}$ is relatively compact.

Let $c=M\left(\left\|x_{0}\right\|+N_{p}\right)+N\left(\left\|x_{1}\right\|+N_{q}\right)$. If

$$
2 N \int_{0}^{T} m(s) d s<\int_{2 c}^{\infty} \frac{1}{W(s)} d s,
$$

then there exists a mild solution of (2.1)-(2.3).

Proof. In order to use Lemma 1.1, we obtain an a priori bound for the solution of the integral equation

$$
\begin{aligned}
x_{\lambda}(t)= & \lambda C(t)\left(x_{0}-p\left(x_{\lambda}\right)\right)+\lambda S(t)\left(x_{1}-q\left(x_{\lambda}\right)\right) \\
& +\lambda \int_{0}^{t} S(t-s) f\left(s, x_{\lambda}(s), x_{\lambda}(a(s))\right) d s
\end{aligned}
$$

for $t \in I$. Using the notations introduced in Section 1 , for $t \in I$ and $\lambda \in(0,1)$ we get

$$
\begin{aligned}
\left\|x_{\lambda}(t)\right\| \leq & M\left(\left\|x_{0}\right\|+N_{p}\right)+N\left(\left\|x_{1}\right\|+N_{q}\right) \\
& +N \int_{0}^{t} m(s) W\left(\left\|x_{\lambda}(s)\right\|+\left\|x_{\lambda}(a(s))\right\|\right) d s .
\end{aligned}
$$

Designating by $\alpha_{\lambda}(t)$ the right hand side above and using the condition $a(t) \leq t$ we obtain

$$
\alpha_{\lambda}^{\prime}(t) \leq N m(t) W\left(2 \alpha_{\lambda}(t)\right)
$$

It follows that

$$
\int_{2 c}^{2 \alpha_{\lambda}(t)} \frac{d s}{W(s)} \leq 2 N \int_{0}^{t} m(s) d s<\int_{2 c}^{\infty} \frac{d s}{W(s)},
$$

which implies that $\alpha_{\lambda}(\cdot)$ is bounded uniformly with respect to $\lambda$, and consequently the set $\left\{x_{\lambda}: \lambda \in(0,1)\right\}$ is bounded in $C(I ; X)$. 
Next, we prove that the operator $\mathcal{T}: C(I ; X) \rightarrow C(I ; X)$ defined by

$$
\begin{array}{r}
\mathcal{T}(x)(t)=C(t)\left(x_{0}-p(x)\right)+S(t)\left(x_{1}-q(x)\right) \\
+\int_{0}^{t} S(t-s) f(s, x(s), x(a(s))) d s
\end{array}
$$

for $t \in I$ is completely continuous. Define $\mathcal{T}_{1}, \mathcal{T}_{2}: C(I ; X) \rightarrow C(I ; X)$ by

$$
\begin{aligned}
\mathcal{T}_{1}(x) & =C(\cdot)\left(x_{0}-p(x)\right)+S(\cdot)\left(x_{1}-q(x)\right), \\
\mathcal{T}_{2}(x)(t) & =\int_{0}^{t} S(t-s) f(s, x(s), x(a(s))) d s, \quad t \in I .
\end{aligned}
$$

From hypothesis $(\mathrm{H}-1)$ it is clear that $\mathcal{T}_{1}$ is completely continuous. Thus, it remains to prove that $\mathcal{T}_{2}$ is completely continuous. The continuity of $\mathcal{T}_{2}$ is a consequence of the Lebesgue dominated convergence theorem. To prove the compactness condition we denote by $B_{r}$ the closed ball in $C(I ; X)$ with center at 0 and radius $r$. We first establish that the set $\mathcal{T}_{2}\left(B_{r}\right)$ is equicontinuous.

We fix $t \in I$ and let $h$ be such that $t+h \in I$. For $x \in B_{r}$, from the definition of $\mathcal{T}_{2}$ it follows that

$$
\begin{aligned}
\left\|\mathcal{T}_{2}(x)(t+h)-\mathcal{T}_{2}(x)(t)\right\| & \\
= & \int_{0}^{t}\|(S(t+h-s)-S(t-s)) f(s, x(s), x(a(s)))\| d s \\
& +\int_{t}^{t+h}\|S(t+h-s) f(s, x(s), x(a(s)))\| d s \\
\leq & M h \int_{0}^{t} m(s) W(\|x(s)\|+\|x(a(s))\|) d s \\
& +N \int_{t}^{t+h} m(s) W(\|x(s)\|+\|x(a(s))\|) d s \\
\leq & M W(2 r) h \int_{0}^{t} m(s) d s+N W(2 r) \int_{t}^{t+h} m(s) d s,
\end{aligned}
$$

which shows that

$$
\left\|\mathcal{T}_{2}(x)(t+h)-\mathcal{T}_{2}(x)(t)\right\| \rightarrow 0, \quad h \rightarrow 0,
$$

uniformly with respect to $x \in B_{r}$.

Next we prove that $\mathcal{T}_{2}\left(B_{r}\right)(t)=\left\{\mathcal{T}_{2} x(t): x \in B_{r}\right\}$ is relatively compact in $X$, for each $t \in I$. We put $U=\{f(t-s, x(t-s), x(a(t-s))): 0 \leq s \leq t$, 
$\left.x \in B_{r}\right\}$. Since

$$
\|y\| \leq m(t-s) W(2 r) \leq m_{T} W(2 r)
$$

for all $y \in U$, and the $\operatorname{map} S(\cdot):[0, T] \rightarrow \mathcal{L}(X)$ is continuous for the operator norm, we infer that for each $\varepsilon>0$ there are $t_{1}, \ldots, t_{n} \in I$ so that for each $0 \leq s \leq t$ and $y \in U$ we can select $t_{i}$ for which

$$
\left\|S(s) y-S\left(t_{i}\right) y\right\| \leq \varepsilon .
$$

Since by hypothesis (H-2) the set $\bigcup_{i=1}^{n} S\left(t_{i}\right) U$ is relatively compact we deduce that the set $\{S(s) y: 0 \leq s \leq t, y \in U\}$ is also relatively compact. The mean value theorem for the Bochner integral ([13]) yields

$$
\mathcal{T}_{2}\left(B_{r}\right)(t) \subseteq t \overline{\operatorname{co}\{S(s) y: 0 \leq s \leq t, y \in U\}},
$$

where co is used to denote the convex hull; this shows the assertion.

Finally, an application of the Ascoli-Arzelà Theorem completes our proof that $\mathcal{T}_{2}\left(B_{r}\right)$ is relatively compact and that $\mathcal{T}_{2}$ is completely continuous.

The existence of a mild solution of the abstract second order nonlocal Cauchy problem (2.1)-(2.3) is now a consequence of Lemma 1.1.

The sine functions that arise in applications are frequently compact. This fact motivates the following corollary.

Corollary 2.1. Assume that the following conditions hold:

(i) $S(t)$ is compact for all $t \geq 0$;

(ii) $p$ and $q$ are bounded and $p$ is completely continuous.

If $c, m$ and $W$ satisfy the assumptions of Theorem 2.1, then there exists a mild solution of (2.1)-(2.3).

From the properties of the abstract Cauchy problem mentioned in the preliminaries we know that if $x_{0}-p(x) \in E$ then the mild solution $x$ is continuously differentiable on $I$ and

$$
\begin{aligned}
x^{\prime}(t)= & A S(t)\left(x_{0}-p(x)\right)+C(t)\left(x_{1}-q(x)\right) \\
& +\int_{0}^{t} C(t-s) f(s, x(s), x(a(s))) d s .
\end{aligned}
$$

Next we study the differentiability of the function $x^{\prime}$. First we consider the following concept of strong solution.

Definition 2.2. A function $x(\cdot): I \rightarrow X$ is a strong solution of (2.1)(2.3) if $x(\cdot) \in W^{2,1}(I ; X)$, equation (2.1) holds for a.e. $t \in I$ and conditions (2.2) and (2.3) are satisfied.

In our results we consider Banach spaces that have the Radon-Nikodým property (abbreviated RNP). We refer to [8] for this matter. 
TheOrem 2.2. Assume that $X$ has the RNP and that the following conditions hold:

$$
\mathcal{R}(p) \subseteq x_{0}+D(A) \text { and } \mathcal{R}(q) \subseteq x_{1}+E
$$

(H-4) For each bounded set $D \subseteq X$, the functions $C(\cdot) f(t, x, y), t \in I$, $x, y \in D$, are uniformly Lipschitz continuous on $I$.

Then each mild solution of (2.1)-(2.3) is a strong solution.

Proof. Let $x(\cdot)$ be a mild solution of (2.1)-(2.3). From (2.6) and (H-3) we get

$$
\begin{aligned}
x^{\prime}(t+s)-x^{\prime}(t)= & (S(t+s)-S(t)) A\left(x_{0}-p(x)\right) \\
& +(C(t+s)-C(t))\left(x_{1}-q(x)\right) \\
& +\int_{0}^{t}(C(t+s-\xi)-C(t-\xi)) f(\xi, x(\xi), x(a(\xi))) d \xi \\
& +\int_{t}^{t+s} C(t+s-\xi) f(\xi, x(\xi), x(a(\xi))) d \xi,
\end{aligned}
$$

which, by (H-4), implies that $x^{\prime}(\cdot)$ is Lipschitz continuous. Since $X$ has the $\mathrm{RNP}$, it follows that $x \in W^{2,1}([0, b] ; X)$. Now, the assertion is a consequence of Proposition 3.3 in [11].

REMARK. Related with this result, we point out that the results in [12] imply that condition (H-4) holds when $\mathcal{R}(f) \subseteq E$ and $f: I \times X \times X \rightarrow E$ takes bounded sets into bounded sets.

In order to discuss the existence of classical solutions we introduce the following concept.

Definition 2.3. A function $x(\cdot): I \rightarrow X$ is a classical solution of problem (2.1)-(2.3) if $x(\cdot)$ is a function of class $C^{2}$ that satisfies equation (2.1) and the initial conditions (2.2) and (2.3).

TheOrem 2.3. Assume that $X$ has the RNP and condition (H-3) holds, together with the following conditions:

(H-5) a is Lipschitz continuous.

(H-6) $\quad f: I \times D \times D \rightarrow X$ is Lipschitz continuous for each bounded $D \subseteq X$.

Then each mild solution of (2.1)-(2.3) is a classical solution.

Proof. Let $x(\cdot)$ be a mild solution of (2.1)-(2.3). From (H-3) it follows that $x$ is a function of class $C^{1}$ and applying (H-5) and (H-6) it is easy to see that the function $h(t)=f(t, x(t), x(a(t)))$ is Lipschitz continuous. The assertion is now a consequence of Theorem 3.1 in [11]. 
In what follows we consider the initial value problem (1.1)-(1.3). To study this problem we always assume that the following conditions hold.

Assumption $\mathrm{A}_{2}$.

(a) $a, b: I \rightarrow I$ are continuous, nondecreasing and for every $t \in I$, $\max \{a(t), b(t)\} \leq t$.

(b) $f: I \times X^{4} \rightarrow X$ satisfies the following Carathéodory conditions:

(i) $f(t, \cdot): X^{4} \rightarrow X$ is continuous for a.e. $t \in I$;

(ii) $f(\cdot, u): I \rightarrow X$ is strongly measurable for each $u \in X^{4}$.

(c) There exists a continuous function $m: I \rightarrow[0, \infty)$ and a continuous nondecreasing function $W:[0, \infty) \rightarrow(0, \infty)$ such that

$$
\left\|f\left(t, x_{1}, x_{2}, x_{3}, x_{4}\right)\right\| \leq m(t) W\left(\sum_{i=1}^{4}\left\|x_{i}\right\|\right), \quad t \in I, x_{i} \in X .
$$

(d) $p, q: C(I ; X)^{2} \rightarrow X$ are continuous.

Definition 2.4. A function $x(\cdot): I \rightarrow X$ is a mild solution of problem (1.1)-(1.3) if: $x(\cdot)$ and $x^{\prime}(\cdot)$ are continuous, the initial conditions (1.2)-(1.3) are satisfied and $x(\cdot)$ satisfies the integral equation

$$
\begin{aligned}
x(t)= & C(t)\left(x_{0}-p\left(x, x^{\prime}\right)\right)+S(t)\left(x_{1}-q\left(x, x^{\prime}\right)\right) \\
& +\int_{0}^{t} S(t-s) f\left(s, x(s), x(a(s)), x^{\prime}(s), x^{\prime}(b(s))\right) d s, \quad t \in I .
\end{aligned}
$$

We observe that if $x$ is a mild solution of (1.1)-(1.3), then $x_{0}-p\left(x, x^{\prime}\right)$ $\in E$. Furthermore, from the already mentioned results of Kisyński [12] we deduce that $D(A)$ is continuously included in $E$ and the operator-valued map $A S(\cdot): I \rightarrow \mathcal{L}(E ; X)$ is continuous. Set $N_{1}=\max _{0 \leq t \leq T}\|A S(t)\|_{\mathcal{L}(E ; X)}$.

THEOREM 2.4. Assume that the following conditions are satisfied:

(H-7) $\quad p, q$ are completely continuous and bounded.

(H-8) One of the following conditions holds:

(a) $x_{0}-p$ is completely continuous and bounded with values in $E$.

(b) $S(t)$ is compact for $t \geq 0$ and $x_{0}-p$ is continuous and bounded with values in $[D(A)]$.

(H-9) $\quad f\left(I \times B^{4}\right)$ is relatively compact for every bounded $B \subset X$.

Let $N_{p}^{1}=\sup \left\{\left\|x_{0}-p(x, y)\right\|_{1}: x, y \in C(I ; X)\right\}$. If

$$
2(M+N) \int_{0}^{T} m(s) d s<\int_{2 c}^{\infty} \frac{d s}{W(s)}
$$

where $c=M\left(\left\|x_{0}\right\|+N_{p}\right)+N_{1} N_{p}^{1}+(M+N)\left(\left\|x_{1}\right\|+N_{q}\right)$, then there exists a mild solution of (1.1)-(1.3). 
Proof. Let $\mathcal{T}: C(I ; X)^{2} \rightarrow C(I ; X)^{2}$ be defined by $\mathcal{T}(x, y)=\left(\mathcal{T}^{1}(x, y)\right.$, $\left.\mathcal{T}^{2}(x, y)\right)$, where

$$
\begin{aligned}
\mathcal{T}^{1}(x, y)(t)= & C(t)\left(x_{0}-p(x, y)\right)+S(t)\left(x_{1}-q(x, y)\right) \\
& +\int_{0}^{t} S(t-s) f(s, x(s), x(a(s)), y(s), y(b(s))) d s, \\
\mathcal{T}^{2}(x, y)(t)= & A S(t)\left(x_{0}-p(x, y)\right)+C(t)\left(x_{1}-q(x, y)\right) \\
& +\int_{0}^{t} C(t-s) f(s, x(s), x(a(s)), y(s), y(b(s))) d s,
\end{aligned}
$$

First, we obtain an a priori bound for the solutions of $z_{\lambda}=\lambda \mathcal{T}\left(z_{\lambda}\right), \lambda \in$ $(0,1)$. For $z_{\lambda}=\left(x_{\lambda}, y_{\lambda}\right), \lambda \in(0,1)$, and $t \in I$ we get

$$
\begin{aligned}
\left\|x_{\lambda}(t)\right\| \leq & M\left(\left\|x_{0}\right\|+N_{p}\right)+N\left(\left\|x_{1}\right\|+N_{q}\right) \\
& +N \int_{0}^{t} m(s) W\left(\left\|x_{\lambda}(s)\right\|+\left\|x_{\lambda}(a(s))\right\|+\left\|y_{\lambda}(s)\right\|+\left\|y_{\lambda}(b(s))\right\|\right) d s \\
= & u_{\lambda}(t),
\end{aligned}
$$

and

$$
\begin{aligned}
\left\|y_{\lambda}(t)\right\| \leq & N_{1} N_{p}^{1}+M\left(\left\|x_{1}\right\|+N_{q}\right) \\
& +M \int_{0}^{t} m(s) W\left(2 u_{\lambda}(s)+\left\|y_{\lambda}(s)\right\|+\left\|y_{\lambda}(b(s))\right\|\right) d s \\
= & v_{\lambda}(t) .
\end{aligned}
$$

Hence

$$
\begin{aligned}
& u_{\lambda}^{\prime}(t) \leq N m(t) W\left(2 u_{\lambda}(t)+2 v_{\lambda}(t)\right), \\
& v_{\lambda}^{\prime}(t) \leq M m(t) W\left(2 u_{\lambda}(t)+2 v_{\lambda}(t)\right),
\end{aligned}
$$

for $t \in[0, T]$. Integrating between 0 and $t$ the sum of (2.7) and (2.8) we obtain

$$
\int_{2 c}^{2\left(u_{\lambda}+v_{\lambda}\right)(t)} \frac{d s}{W(s)} \leq 2(M+N) \int_{0}^{t} m(s) d s<\int_{2 c}^{\infty} \frac{d s}{W(s)},
$$

which implies that the set $\left\{\left(x_{\lambda}, y_{\lambda}\right): \lambda \in(0,1)\right\}$ is bounded in $C(I ; X)^{2}$.

It is easy to prove that $\mathcal{T}$ is continuous. Moreover, repeating the arguments employed in the proof of Theorem 2.1 we infer that $\mathcal{T}^{1}$ is completely continuous. Further, if (a) or (b) holds, then $A S(\cdot)\left(x_{0}-p\right)$ is completely continuous, which together with (H-7) and (H-9) permits us to conclude that $\mathcal{T}^{2}$ is completely continuous.

The existence of a mild solution for (1.1)-(1.3) is now a consequence of Lemma 1.1. The proof is complete. 
We conclude this section with some regularity results for the mild solutions of the initial value problem (1.1)-(1.3). First we introduce some definitions.

Definition 2.5. A function $x(\cdot): I \rightarrow X$ is a strong solution of (1.1)(1.3) if $x(\cdot) \in W^{2,1}(I ; X)$, equation (1.1) is satisfied for a.e. $t \in I$ and conditions (1.2) and (1.3) are satisfied.

Definition 2.6. A function $x(\cdot): I \rightarrow X$ is a classical solution of (1.1)-(1.3) if $x(\cdot)$ is a function of class $C^{2}$ that satisfies equation (1.1) and the initial conditions (1.2) and (1.3).

To prove Theorems 2.5 and 2.6 that follow we argue as in the proofs of Theorems 2.2 and 2.3, respectively. For this reason we omit the details.

Theorem 2.5. Assume that $X$ has the RNP, (H-3) holds, and moreover $(\mathrm{H}-4)^{\prime} \quad$ For each bounded set $D \subseteq X$, the functions $C(\cdot) f\left(t, x_{1}, x_{2}, x_{3}, x_{4}\right)$, $t \in I, x_{i} \in D$, are uniformly Lipschitz continuous on $I$.

Then each mild solution of (1.1)-(1.3) is a strong solution.

TheOrem 2.6. Assume that $X$ has the $R N P,(\mathrm{H}-3)$ is fulfilled, and the following conditions hold:

(H-10) $\quad a, b$ are Lipschitz continuous.

(H-11) $\quad f: I \times D^{4} \rightarrow X$ is Lipschitz continuous for each bounded $D \subseteq X$.

Then each mild solution of (1.1)-(1.3) is a classical solution.

3. Existence of global solutions. In this section we study the problem (2.1)-(2.3) on the interval $I=[0, \infty)$. We suppose that Assumption $\mathrm{A}_{1}$ of Section 2 is satisfied on $I$ and that $M$ and $N$ are positive constants such that $\|C(t)\| \leq M$ and $\|S(t)\| \leq N$ for all $t \geq 0$. To generalize the arguments employed in the previous section to include continuous functions defined on $[0, \infty)$ we need an appropriate version of the Ascoli-Arzelà theorem. For this reason we consider two cases. First, we study the existence of solutions in the space of continuous functions with weight; next, we establish the existence of asymptotically almost periodic solutions.

Let $g:[0, \infty) \rightarrow(0, \infty)$ be a continuous function such that $g(t) \rightarrow \infty$ as $t \rightarrow \infty$. For simplicity we also assume that $g$ is increasing and $g(0)=1$. Next, $C_{g}^{0}(X)$ stands for the Banach space formed by the continuous functions $x:[0, \infty) \rightarrow X$ such that $x(t) / g(t) \rightarrow 0$ as $t \rightarrow \infty$, endowed with the norm

$$
\|x\|_{g}=\sup _{t \geq 0} \frac{\|x(t)\|}{g(t)}
$$

We also denote by $C_{0}(X)$ the Banach space formed by the continuous functions $x:[0, \infty) \rightarrow X$ that vanish at infinity. It is well known that each 
function in $C_{0}(X)$ is uniformly continuous. We will frequently use the fact that $1 / g$ is uniformly continuous. We recall the following result on compactness in $C_{0}(X)$.

Lemma 3.1. A set $B \subseteq C_{0}(X)$ is relatively compact if, and only if, the following conditions are fulfilled:

(a) $B$ is equicontinuous.

(b) $x(t) \rightarrow 0$ as $t \rightarrow \infty$, uniformly for $x \in B$.

(c) The orbits $B(t)$ are relatively compact in $X$ for all $t \geq 0$.

Next, without any danger of confusion, we abbreviate our notations by writing $u(t)=(x(t), x(a(t)))$, for a specified function $x$.

TheOREM 3.1. Assume that the following conditions are satisfied:

(a) $p, q: C_{g}^{0}(X) \rightarrow X$ are continuous, bounded and $p$ is completely continuous.

(b) $S(t) q: C_{g}^{0}(X) \rightarrow X$ is completely continuous for each $t \geq 0$.

(c) For each $t \in I, t^{\prime} \leq t$ and a constant $L \geq 0$ the set $\left\{S\left(t^{\prime}\right) f(s, x, y)\right.$ : $0 \leq s \leq t,\|x\|,\|y\| \leq L\}$ is relatively compact.

(d) For every constant $L \geq 0,(1 / g(t)) \int_{0}^{t} m(s) W(L g(s)) d s \rightarrow 0$ as $t \rightarrow \infty$.

(e) $2 N \int_{0}^{\infty} m(s) d s<\int_{2 c}^{\infty}(1 / W(s)) d s$, where $c=M\left(\left\|x_{0}\right\|+N_{p}\right)+N\left(\left\|x_{1}\right\|\right.$ $\left.+N_{q}\right)$.

Then there exists a mild solution $x \in C_{g}^{0}(X)$ of $(2.1)-(2.3)$.

Proof. For each $x \in C_{g}^{0}(X)$ we define $\mathcal{T}(x)(t)$ by means of (2.5). Hence,

$$
\begin{aligned}
\|\mathcal{T}(x)(t)\| \leq & M\left(\left\|x_{0}\right\|+N_{p}\right)+N\left(\left\|x_{1}\right\|+N_{q}\right) \\
& +N \int_{0}^{t} m(s) W(\|x(s)\|+\|x(a(s))\|) d s .
\end{aligned}
$$

Since $\|x(s)\| \leq\|x\|_{g} g(s)$, applying condition (d) it follows that

$$
\begin{aligned}
\frac{\|\mathcal{T}(x)(t)\|}{g(t)} \leq & \frac{M}{g(t)}\left(\left\|x_{0}\right\|+N_{p}\right) \\
& +\frac{N}{g(t)}\left(\left\|x_{1}\right\|+N_{q}\right)+\frac{N}{g(t)} \int_{0}^{t} m(s) W\left(2\|x\|_{g} g(s)\right) d s
\end{aligned}
$$

converges to zero as $t \rightarrow \infty$. This shows that $\mathcal{T}$ is a well defined map from $C_{g}^{0}(X)$ into $C_{g}^{0}(X)$. To prove the continuity of $\mathcal{T}$, we take a sequence $\left(x_{n}\right)_{n}$ which converges to $x$ in $C_{g}^{0}(X)$. It is clear that $f\left(s, u_{n}(s)\right) \rightarrow f(s, u(s))$ a.e. and $n \rightarrow \infty$,

$$
\left\|f\left(s, u_{n}(s)\right)\right\| \leq m(s) W(L g(s)),
$$

for some constant $L \geq 0$. Since the right hand side is integrable on $[0, t]$ we conclude that $\mathcal{T}\left(x_{n}\right)(t) \rightarrow \mathcal{T}(x)(t)$ as $n \rightarrow \infty$. It is also clear that the 
convergence is uniform for $t$ in bounded intervals. Furthermore, given $\varepsilon>0$, applying (a) and (d) we can choose $t_{0}$ so that

$$
\begin{aligned}
\frac{1}{g(t)}\left\|\mathcal{T}\left(x_{n}\right)(t)-\mathcal{T}(x)(t)\right\| \leq & \frac{M}{g(t)}\left\|p\left(x_{n}\right)-p(x)\right\|+\frac{N}{g(t)}\left\|q\left(x_{n}\right)-q(x)\right\| \\
& +\frac{2 N}{g(t)} \int_{0}^{t} m(s) W(L g(s)) d s \leq \varepsilon
\end{aligned}
$$

for $t \geq t_{0}$. This implies that $\mathcal{T}\left(x_{n}\right) \rightarrow \mathcal{T}(x)$ as $n \rightarrow \infty$ in the space $C_{g}^{0}(X)$. Consequently, $\mathcal{T}$ is a continuous map.

Now, for $0<\lambda<1$ let $x_{\lambda} \in C_{g}^{0}(X)$ be such that $\lambda \mathcal{T}\left(x_{\lambda}\right)=x_{\lambda}$. Proceeding as above we can estimate

$$
\begin{aligned}
\left\|x_{\lambda}(t)\right\| \leq & M\left(\left\|x_{0}\right\|+N_{p}\right)+N\left(\left\|x_{1}\right\|+N_{q}\right) \\
& \left.+N \int_{0}^{t} m(s) W\left(\left\|x_{\lambda}(s)\right\|+\| x_{\lambda}(s)\right) \|\right) d s .
\end{aligned}
$$

If $\alpha_{\lambda}(t)$ denotes the right hand side, repeating the argument used in the proof of Theorem 2.1 we conclude that the set $\left\{\alpha_{\lambda}(t): 0<\lambda<1, t \geq 0\right\}$ is bounded, which in turn implies that $\left\{\left\|x_{\lambda}(t)\right\|: 0<\lambda<1, t \geq 0\right\}$ and $\left\{\left\|x_{\lambda}\right\|_{g}: 0<\lambda<1\right\}$ are bounded.

Finally, we show that $\mathcal{T}$ is completely continuous. Since we have already established that $\mathcal{T}$ is continuous, it only remains to prove that $\mathcal{T}(B)$ is relatively compact in $C_{g}^{0}(X)$ for every bounded set $B$. Thus, we need to verify that the functions $(1 / g) \mathcal{T}(x), x \in B$, satisfy the conditions of Lemma 3.1. To prove that $(1 / g) \mathcal{T}(x), x \in B$, are equicontinuous, we observe first that the set of functions $\left\{(1 / g)\left[C(\cdot)\left(x_{0}-p(x)\right)+S(\cdot)\left(x_{1}-q(x)\right)\right]: x \in B\right\}$ is equicontinuous, because $p$ is completely continuous, $q$ is bounded and $1 / g$ is uniformly continuous. Moreover, for $x \in B$ and $h \geq 0$, there is a constant $L \geq 0$ for which

$$
\begin{aligned}
\| \int_{0}^{t+h} S(t+h-s) & f(s, u(s)) d s-\int_{0}^{t} S(t-s) f(s, u(s)) d s \| \\
\leq & \left\|\int_{0}^{t}[S(t+h-s)-S(t-s)] f(s, u(s)) d s\right\| \\
& +\left\|\int_{t}^{t+h} S(t+h-s) f(s, u(s)) d s\right\| \\
\leq & M h \int_{0}^{t} m(s) W(\operatorname{Lg}(s)) d s+N \int_{t}^{t+h} m(s) W(\operatorname{Lg}(s)) d s .
\end{aligned}
$$


This shows that

$$
\frac{1}{g(t+h)} \mathcal{T}(x)(t+h)-\frac{1}{g(t)} \mathcal{T}(x)(t) \rightarrow 0 \quad \text { as } h \rightarrow 0,
$$

independently of $x \in B$. The same argument and condition (d) also permit us to state that $(1 / g(t)) \mathcal{T}(x)(t) \rightarrow 0$ as $t \rightarrow \infty$, uniformly with respect to $x \in B$.

Finally, applying our hypotheses (a), (b), (c) and proceeding as in the proof of Theorem 2.1 we derive that the set $\{\mathcal{T}(x)(t): x \in B\}$ is relatively compact in $X$, for each $t \geq 0$. Consequently, $\{\mathcal{T}(x) / g: x \in B\}$ is relatively compact in $C_{0}(X)$ and $\mathcal{T}(B)$ is relatively compact in $C_{g}^{0}(X)$.

We now study the existence of asymptotically almost periodic solutions of (2.1)-(2.3). For the basic information about almost periodic and asymptotically almost periodic functions we refer to [20].

In particular, we denote by $\operatorname{AP}(X)$ (resp. $\operatorname{AAP}(X))$ the space formed by the functions $x:[0, \infty) \rightarrow X$ which are almost periodic (resp. asymptotically almost periodic), endowed with the norm of uniform convergence. We will also utilize the properties of almost periodic cosine functions and almost periodic sine functions. We refer to [6] for the characterization of almost periodic cosine functions and to [10] for similar properties of almost periodic sine functions. The following lemma ([20]) is a result of Ascoli-Arzelà type for $\operatorname{AAP}(X)$.

LEMMA 3.2. Let $B \subseteq \operatorname{AAP}(X)$ have the following properties:

(a) $B$ is equicontinuous uniformly on $[0, \infty)$.

(b) $B$ is equi-asymptotically almost periodic, that is, for every $\varepsilon>0$ there are $T_{\varepsilon} \geq 0$ and a relatively dense set $P_{\varepsilon}$ in $[0, \infty)$ such that

$$
\|x(t+\tau)-x(t)\| \leq \varepsilon, \quad x \in B, t \geq T_{\varepsilon}, \tau \in P_{\varepsilon} .
$$

(c) For each $t \geq 0, B(t)$ is relatively compact in $X$.

Then $B$ is relatively compact in $\operatorname{AAP}(X)$.

To establish our result we need the following property of compactness of integrable functions.

LEMMA 3.3. Let $B \subseteq \mathcal{L}^{1}([0, \infty) ; X)$ satisfy:

(a) $B$ is equiintegrable at $\infty$, that is, $\int_{L}^{\infty}\|x(s)\| d s \rightarrow 0$ as $L \rightarrow \infty$, uniformly for $x \in B$.

(b) For each $t \geq 0$, the set $\{x(s): x \in B, 0 \leq s \leq t\}$ is relatively compact in $X$.

If $F:[0, \infty) \rightarrow \mathcal{L}(X)$ is a strongly continuous and uniformly bounded operator-valued function, then there is a compact set $U \subseteq X$ such that $\int_{0}^{t} F(s) x(s) d s \in U$ for all $x \in B$ and $0 \leq t \leq \infty$. 
Proof. For each $\varepsilon>0$ we can select $L \geq 0$ such that $\left\|\int_{L}^{\infty} F(s) x(s) d s\right\|$ $\leq \varepsilon$ for all $x \in B$. By (b) there is a compact set $U_{0} \subseteq X$ such that $x(s) \in U_{0}$ for all $0 \leq s \leq L$ and $x \in B$. Since the map $[0, L] \times U_{0} \rightarrow X,(s, x) \mapsto F(s) x$, is continuous we infer that $\{F(s) x(s): 0 \leq s \leq L, x \in B\}$ is relatively compact, and the mean value theorem for the Bochner integral ([13]) shows that the set $\left\{\int_{0}^{L} F(s) x(s) d s: x \in B\right\}$ has the same property. The decomposition

$$
\int_{0}^{t} F(s) x(s) d s=\int_{0}^{L} F(s) x(s) d s+\int_{L}^{t} F(s) x(s) d s
$$

completes the proof.

Now we combine these results to establish the following property of mild solutions of the abstract Cauchy problem. In this statement we denote by $y_{x}:[0, \infty) \rightarrow X$ the function defined by

$$
y_{x}(t)=\int_{0}^{t} S(t-s) x(s) d s, \quad t \geq 0 .
$$

Proposition 3.1. Assume that the sine function $S(\cdot)$ is almost periodic. Let $B \subseteq \mathcal{L}^{1}([0, \infty) ; X)$ satisfy conditions (a), (b) of Lemma 3.3 and the following condition of equiintegrability: for each $t \geq 0, \int_{t}^{t+h}\|x(s)\| d s \rightarrow 0$ as $h \rightarrow 0$, uniformly for $x \in B$. Then the set $\left\{y_{x}: x \in B\right\}$ is relatively compact in $\operatorname{AAP}(X)$.

Proof. We can write

$$
\begin{aligned}
y_{x}(t)= & S(t) \int_{0}^{t} C(s) x(s) d s-C(t) \int_{0}^{t} S(s) x(s) d s \\
= & S(t) \int_{0}^{\infty} C(s) x(s) d s-S(t) \int_{t}^{\infty} C(s) x(s) d s \\
& -C(t) \int_{0}^{\infty} S(s) x(s) d s+C(t) \int_{t}^{\infty} S(s) x(s) d s .
\end{aligned}
$$

From the properties of almost periodic sine functions ([10]) we deduce that the first and third terms on the right hand side define almost periodic functions, while the second and fourth terms are functions that vanish at $\infty$. We infer that $y_{x} \in \operatorname{AAP}(X)$. Furthermore, Lemma 3.3 shows that the integrals $\int_{0}^{\infty} C(s) x(s) d s$ and $\int_{0}^{\infty} S(s) x(s) d s, x \in B$, lie in a compact set. This implies that the set formed by the functions $S(\cdot) \int_{0}^{\infty} C(s) x(s) d s-$ $C(\cdot) \int_{0}^{\infty} S(s) x(s) d s, x \in B$, is relatively compact in $\operatorname{AP}(X)$. Moreover, employing the properties of $B$ and Lemma 3.3 it is not difficult to see that the set formed by the functions $S(t) \int_{t}^{\infty} C(s) x(s) d s-C(t) \int_{t}^{\infty} S(s) x(s) d s$, 
$x \in B$, satisfies the conditions of Lemma 3.1 , so it is relatively compact in $C_{0}(X)$. Collecting these results yields the assertion.

Now we are in a position to establish the main result.

Theorem 3.2. Assume that $S(\cdot)$ is almost periodic and that the following conditions hold:

(a) $p, q: \operatorname{AAP}(X) \rightarrow X$ are completely continuous and bounded.

(b) For each $t>0$ and each constant $L \geq 0$ the set $\{f(s, x, y): 0 \leq s \leq t$, $\|x\|,\|y\| \leq L\}$ is relatively compact.

(c) $2 N \int_{0}^{\infty} m(s) d s<\int_{2 c}^{\infty}(1 / W(s)) d s$, where $c=M\left(\left\|x_{0}\right\|+N_{p}\right)+$ $N\left(\left\|x_{1}\right\|+N_{q}\right)$.

Then there exists a mild solution $x \in \operatorname{AAP}(X)$ of $(2.1)-(2.3)$.

Proof. For each $x \in \operatorname{AAP}(X)$ we define $\mathcal{T}(x)(t)$ by means of (2.5). From our hypotheses and proceeding as in the proof of Proposition 3.1 we deduce easily that $\mathcal{T}(x) \in \operatorname{AAP}(X)$ for each $x \in \operatorname{AAP}(X)$. To prove the continuity of $\mathcal{T}: \operatorname{AAP}(X) \rightarrow \operatorname{AAP}(X)$ we take a sequence $\left(x_{n}\right)_{n}$ in $\operatorname{AAP}(X)$ that converges to $x$. Clearly $S(t-s) f\left(s, u_{n}(s)\right) \rightarrow S(t-s) f(s, u(s))$ as $n \rightarrow \infty$, for a.e. $s \in[0, t]$. Since

$$
\left\|S(t-s) f\left(s, u_{n}(s)\right)-S(t-s) f(s, u(s))\right\| \leq 2 N m(s) W(L)
$$

for some constant $L \geq 0$, and the right hand side is integrable on $[0, \infty)$, we conclude that $\mathcal{T}\left(x_{n}\right)(t) \rightarrow \mathcal{T}(x)(t)$ as $n \rightarrow \infty$, and that the convergence is uniform on $[0, \infty)$. Hence $\mathcal{T}$ is continuous.

Next, for $\lambda \in(0,1)$, let $x_{\lambda} \in \operatorname{AAP}(X)$ be such that $\lambda \mathcal{T}\left(x_{\lambda}\right)=x_{\lambda}$. Proceeding as in the proof of Theorem 2.1 we conclude that $\left\{x_{\lambda}: \lambda \in(0,1)\right\}$ is bounded.

Finally, we show that $\mathcal{T}$ is completely continuous. We take a bounded set $B \subseteq \operatorname{AAP}(X)$. Since $p$ and $q$ are completely continuous, it is clear that the set $\left\{C(\cdot)\left(x_{0}-p(x)\right)+S(\cdot)\left(x_{1}-q(x)\right): x \in B\right\}$ is relatively compact in $\operatorname{AAP}(X)$. In addition, since the functions $f(s, u(s)), x \in B$, satisfy the hypotheses of Proposition 3.1 we infer that the set $\left\{z_{x}: x \in B\right\}$, where $z_{x}(t)=\int_{0}^{t} S(t-s) f(s, u(s)) d s$, is relatively compact in $\operatorname{AAP}(X)$. This completes the proof.

We say that the sine function $S(\cdot)$ is uniformly almost periodic if $S$ : $\mathbb{R} \rightarrow \mathcal{L}(X)$ is almost periodic. For instance, every periodic sine function is uniformly almost periodic. Arguing as in the proof of Theorem 3.2 we can establish the following results. We omit the proofs.

Theorem 3.3. Assume that $S(\cdot)$ is uniformly almost periodic and that the following conditions hold: 
(a) $p, q: \operatorname{AAP}(X) \rightarrow X$ are continuous and bounded and $p$ is completely continuous.

(b) $S(t) q: \operatorname{AAP}(X) \rightarrow X$ is completely continuous for each $t \geq 0$.

(c) For each $0 \leq t^{\prime} \leq t$ and each constant $L \geq 0$ the set $\left\{S\left(t^{\prime}\right) f(s, x, y)\right.$ : $0 \leq s \leq t,\|x\|,\|y\| \leq L\}$ is relatively compact.

(d) $2 N \int_{0}^{\infty} m(s) d s<\int_{2 c}^{\infty}(1 / W(s)) d s$, where $c=M\left(\left\|x_{0}\right\|+N_{p}\right)+$ $N\left(\left\|x_{1}\right\|+N_{q}\right)$.

Then there exists a mild solution $x \in \operatorname{AAP}(X)$ of (2.1)-(2.3).

Corollary 3.1. Assume that $S(\cdot)$ is compact and uniformly almost periodic and that the following conditions hold:

(a) $p, q: \operatorname{AAP}(X) \rightarrow X$ are continuous and bounded and $p$ is completely continuous.

(b) $2 N \int_{0}^{\infty} m(s) d s<\int_{2 c}^{\infty}(1 / W(s)) d s$, where $c=M\left(\left\|x_{0}\right\|+N_{p}\right)+$ $N\left(\left\|x_{1}\right\|+N_{q}\right)$.

Then there exists a mild solution $x \in \operatorname{AAP}(X)$ of (2.1)-(2.3).

Next we consider a situation where the derivative of a mild solution $x \in \operatorname{AAP}(X)$ is also asymptotically almost periodic. We need the following property of almost periodic sine functions.

LEMma 3.4. Assume that $S(\cdot)$ is almost periodic. Then for all $x \in X$ the function $S(\cdot) x$ is almost periodic for the norm in E. Furthermore, if $x:[0, \infty) \rightarrow X$ is integrable then the function $y:[0, \infty) \rightarrow E$ given by

$$
y(t)=\int_{0}^{t} S(s) x(s) d s
$$

is continuous with range relatively compact in $E$.

Proof. Since $C(\cdot) x$ and $S(\cdot) x$ are almost periodic it follows that $(C(\cdot) x$, $S(\cdot) x)$ is almost periodic in $X \times X$. Thus, given $\varepsilon>0$, there is a relatively dense set $P_{\varepsilon}$ such that

$$
\|C(t+\tau) x-C(t) x\|+\|S(t+\tau) x-S(t) x\| \leq \varepsilon, \quad t \geq 0, \tau \in P_{\varepsilon} .
$$

Using the fact that $C(\cdot)$ is uniformly bounded and

$$
C(t+s) x=C(t) C(s) x+A S(t) S(s) x
$$

we obtain

$$
\begin{aligned}
& \|S(t+\tau) x-S(t) x\|_{1} \\
& \quad=\|S(t+\tau) x-S(t) x\|+\sup _{0 \leq h \leq 1}\|A S(h)[S(t+\tau) x-S(t) x]\| \\
& \quad \leq \varepsilon+\sup _{0 \leq h \leq 1}\|C(t+h+\tau) x-C(h) C(t+\tau) x+C(t) C(h) x-C(t+h) x\|
\end{aligned}
$$




$$
\begin{aligned}
& \leq \varepsilon+\sup _{0 \leq h \leq 1}(\|C(h)\|\|C(t+\tau) x-C(t) x\|+\|C(t+h+\tau) x-C(t+h) x\|) \\
& \leq(M+2) \varepsilon
\end{aligned}
$$

for all $t \geq 0$ and $\tau \in P_{\varepsilon}$, which proves the first assertion.

Moreover, in the introduction we have already mentioned that $y(\cdot)$ is a continuous $E$-valued function. From (3.1) we infer that $S(\cdot) x(\cdot) \in$ $\mathcal{L}^{1}([0, \infty) ; E)$, which allows us to complete the proof with the same argument used in the proof of Lemma 3.3.

The following result is a consequence of the previous property.

Corollary 3.2. Assume that the conditions of Theorem 3.2 hold, $x_{0}-$ $p(x) \in E$, and $A S(\cdot) y$ is uniformly bounded on $[0, \infty)$ for each $y \in E$. Let $x$ be a mild solution of (2.1)-(2.3). Then $x^{\prime}$ is also asymptotically almost periodic.

Proof. From the uniform boundedness principle it follows that the operator family $A S(t): E \rightarrow X, t \geq 0$, is uniformly bounded. This implies that $A S(\cdot) y$ is uniformly continuous on $[0, \infty)$ for each $y \in E$. In fact,

$$
\|A S(t+h) y-A S(t) y\| \leq\|A S(t)\|\|(C(h)-I) y\|+\|C(t)\|\|A S(h) y\|
$$

converges to zero as $h \rightarrow 0$, uniformly with respect to $t \geq 0$.

Moreover, from (1.9) we can write

$$
x^{\prime}(t)=A S(t)\left(x_{0}-p(x)\right)+C(t)\left(x_{1}-q(x)\right)+\int_{0}^{t} C(t-s) f(s, u(s)) d s .
$$

Since $A S(t)\left(x_{0}-p(x)\right)$ is the derivative of $C(t)\left(x_{0}-p(x)\right)$, the previous remark and Theorem 5.2 of [20] imply that $A S(t)\left(x_{0}-p(x)\right)$ is almost periodic. Consequently, the function $A S(t)\left(x_{0}-p(x)\right)+C(t)\left(x_{1}-q(x)\right)$ is almost periodic.

It remains to prove that the function given by

$$
y(t)=\int_{0}^{t} C(t-s) f(s, u(s)) d s
$$

is asymptotically almost periodic. By Theorem 5.2 of [20], it is sufficient to show that $y$ is uniformly continuous. For this purpose we decompose

$$
\begin{aligned}
y(t+h)-y(t)= & \int_{0}^{t}[C(t+h-s)-C(t-s)] f(s, u(s)) d s \\
& +\int_{t}^{t+h} C(t+h-s) f(s, u(s)) d s
\end{aligned}
$$




$$
\begin{aligned}
= & (C(h)-I) y(t)+A S(h) \int_{0}^{t} S(t-s) f(s, u(s)) d s \\
& +\int_{t}^{t+h} C(t+h-s) f(s, u(s)) d s .
\end{aligned}
$$

Since $f(s, u(s))$ is integrable on $[0, \infty)$, the range of $y(\cdot)$ is relatively compact, which implies that $(C(h)-I) y(t) \rightarrow 0$ as $h \rightarrow 0$, uniformly for $t \geq 0$. The same argument shows that the third term of the above expression converges to zero as $h \rightarrow 0$, uniformly with respect to $t \geq 0$. Similarly, employing the preceding lemma we find that the values $\int_{0}^{t} S(t-s) f(s, u(s)) d s, t \geq 0$, lie in a relatively compact subset of $E$ and, from the introduction, we conclude that $A S(h) \int_{0}^{t} S(t-s) f(s, u(s)) d s \rightarrow 0$ as $h \rightarrow 0$, uniformly with respect to $t \geq 0$. This completes the proof.

4. Applications. The one-dimensional wave equation modelled as an abstract Cauchy problem has been extensively studied (see for example [19]). In this section we illustrate some of our results by means of the wave equation. First we introduce some technical remarks.

On $X=L^{2}([0, \pi])$ we consider the operator $A f(\xi)=f^{\prime \prime}(\xi)$ with domain

$$
D(A)=\left\{f(\cdot) \in H^{2}(0, \pi): f(0)=f(\pi)=0\right\} .
$$

It is well known that $A$ is the generator of a strongly continuous cosine function $(C(t))_{t \in \mathbb{R}}$ on $X$. Furthermore, $A$ has discrete spectrum, the eigenvalues are $-n^{2}, n \in \mathbb{N}$, with corresponding normalized eigenvectors $z_{n}(\xi):=$ $\sqrt{2 / \pi} \sin (n \xi)$ and the following conditions hold:

(a) $\left\{z_{n}: n \in \mathbb{N}\right\}$ is an orthonormal basis of $X$.

(b) If $\varphi \in D(A)$ then $A \varphi=-\sum_{n=1}^{\infty} n^{2}\left\langle\varphi, z_{n}\right\rangle z_{n}$.

(c) For every $\varphi \in X, C(t) \varphi=\sum_{n=1}^{\infty} \cos (n t)\left\langle\varphi, z_{n}\right\rangle z_{n}$. Consequently, $\|C(t)\|=1$ for every $t \in \mathbb{R}$.

(d) The associated sine function is given by

$$
S(t) \varphi=\sum_{n=1}^{\infty} \frac{\sin (n t)}{n}\left\langle\varphi, z_{n}\right\rangle z_{n} .
$$

It is clear from this expression that $S(\cdot)$ is $2 \pi$-periodic, compact and $\|S(t)\|=1$ for every $t \in \mathbb{R}$.

(e) If $G$ denotes the group of translations on $X$ defined by $G(t) x(\xi)=$ $\widetilde{x}(\xi+t)$, where $\widetilde{x}$ is the extension of $x$ with period $2 \pi$, then $C(t)=$ $\frac{1}{2}(G(t)+G(-t))$. Hence it follows (see [9]) that $A=B^{2}$, where $B$ is the infinitesimal generator of the group $G$ and $E=\left\{x \in H^{1}(0, \pi)\right.$ : $x(0)=x(\pi)=0\}$. 
Now we consider the boundary value problem

$$
\begin{aligned}
\frac{\partial^{2}}{\partial t^{2}} w(\xi, t) & =\frac{\partial^{2}}{\partial \xi^{2}} w(\xi, t)+F(\xi, t, w(\xi, t), w(\xi, a(t))), \\
w(0, t) & =w(\pi, t)=0, \quad t \in I,
\end{aligned}
$$

where the function $F:[0, \pi] \times I \times \mathbb{R}^{2} \rightarrow \mathbb{R}$ satisfies the following Carathéodory conditions:

(a) $F(\xi, t, \cdot): \mathbb{R}^{2} \rightarrow \mathbb{R}$ is continuous for a.e. $\xi \in[0, \pi], t \in I$.

(b) For every $w_{1}, w_{2} \in \mathbb{R}$ the function $F\left(\cdot, w_{1}, w_{2}\right):[0, \pi] \times I \rightarrow \mathbb{R}$ is measurable.

(c) There exists a positive continuous function $\eta$ on $[0, \pi] \times I$ such that

$$
\left|F\left(\xi, t, w_{1}, w_{2}\right)\right| \leq \eta(\xi, t)\left(\left|w_{1}\right|+\left|w_{2}\right|\right) .
$$

Associated to problem (4.1)-(4.2), we consider the following nonlocal initial conditions:

$$
\begin{aligned}
& w(\xi, 0)+\int_{0}^{T} P(w(\cdot, s))(\xi) d \mu(s)=x_{0}(\xi), \xi \in[0, \pi], \\
& \frac{\partial w(\xi, 0)}{\partial t}+\int_{0}^{T} Q(w(\cdot, s))(\xi) d \nu(s)=x_{1}(\xi), \quad \xi \in[0, \pi],
\end{aligned}
$$

where $\mu, \nu$ are real functions of bounded variation on $I$. We also assume that $P, Q: X \rightarrow X$ are bounded continuous operators and that $P$ is completely continuous. Let $p, q: C_{b}(I ; X) \rightarrow X$ be the maps defined by

$$
\begin{aligned}
& p(x)=\int_{0}^{T} P(x(s)) d \mu(s), \\
& q(x)=\int_{0}^{T} Q(x(s)) d \nu(s),
\end{aligned}
$$

where $C_{b}(I ; X)$ denotes the space of bounded continuous functions from $I$ into $X$ endowed with the norm of uniform convergence. It is not difficult to see that $p, q$ are continuous and bounded and that $p$ is completely continuous.

Under the previous conditions, problem (4.1)-(4.4) can be modelled as (2.1)-(2.3), where $f(t, x, y)(\xi)=F(\xi, t, x(\xi), y(\xi))$ satisfies Assumption $\mathrm{A}_{1}$. Moreover, if $x, y \in X$ then

$$
\|f(t, x, y)\| \leq\left(\int_{0}^{\pi}|F(\xi, t, x(\xi), y(\xi))|^{2} d \xi\right)^{1 / 2}
$$




$$
\begin{aligned}
& \leq\left(\int_{0}^{\pi} \eta^{2}(\xi, t)(|x(\xi)|+|y(\xi)|)^{2} d \xi\right)^{1 / 2} \\
& \leq \max _{0 \leq \xi \leq \pi} \eta(\xi, t)(\|x\|+\|y\|) .
\end{aligned}
$$

Since $S(\cdot)$ is compact, in the case $T<\infty$, from Corollary 2.1 we obtain existence of a mild solution of (4.1)-(4.4).

For $T=\infty$, we consider first the existence of asymptotically almost periodic solutions. In this case we consider $p, q$ defined on $\operatorname{AAP}(X)$. Since $S(\cdot)$ is compact and periodic, Corollary 3.1 shows that if $\int_{0}^{\infty} \max _{0 \leq \xi \leq \pi} \eta(\xi, t) d t$ $<\infty$, then there exists an asymptotically almost periodic solution of (4.1)(4.4).

Finally, we consider the existence of $g$-bounded solutions on $[0, \infty)$. We assume that $g$ satisfies the conditions specified at the beginning of Section 3 . Since $P$ and $Q$ are bounded operators, the maps $p, q$ are well defined by (4.5) and (4.6), respectively, on $C_{g}^{0}(X)$ and they are also bounded. Furthermore, applying the Lebesgue dominated convergence theorem we deduce that $p, q$ are continuous. Moreover, if $V_{\mu}(t)$ denotes the variation of $\mu$ on $[t, \infty)$, then $V_{\mu}(n) \rightarrow 0$ as $n \rightarrow \infty$ and

$$
\left\|\int_{n}^{\infty} P(x(s)) d s\right\| \leq N_{P} V_{\mu}(n) .
$$

From this it follows easily that $p$ is completely continuous. We assume that the following conditions are fulfilled:

(a) $\int_{0}^{\infty} \max _{0 \leq \xi \leq \pi} \eta(\xi, t) d t<\infty$;

(b) $\frac{1}{g(t)} \int_{0}^{t} \max _{0 \leq \xi \leq \pi} \eta(\xi, s) g(s) d s \rightarrow 0$ as $t \rightarrow \infty$.

Under these conditions, by Theorem 3.1, there exists a mild solution $x \in C_{g}^{0}(X)$ of $(4.1)-(4.4)$.

\section{References}

[1] L. Byszewski, Theorems about the existence and uniqueness of solutions of a semilinear evolution nonlocal Cauchy problem, J. Math. Anal. Appl. 162 (1991), 494-505.

[2] —, Existence, uniqueness and asymptotic stability of solutions of abstract nonlocal Cauchy problems, Dynam. Systems Appl. 5 (1996), 595-605.

[3] L. Byszewski and H. Akca, Existence of solutions of a semilinear functional-differential evolution nonlocal problem, Nonlinear Anal. 34 (1998), 65-72.

[4] -, - , On a mild solution of a semilinear functional-differential evolution nonlocal problem, J. Appl. Math. Stochastic Anal. 10 (1997), 265-271. 
[5] L. Byszewski and V. Lakshmikantham, Theorem about the existence and uniqueness of a solution of a nonlocal abstract Cauchy problem in a Banach space, Appl. Anal. 40 (1991), 11-19.

[6] I. Cioranescu, Characterizations of almost periodic strongly continuous cosine operator functions, J. Math. Anal. Appl. 116 (1986), 222-229.

[7] K. Deimling, Nonlinear Functional Analysis, Springer, Berlin, 1985.

[8] J. Diestel and J. J. Uhl Jr., Vector Measures, Math. Surveys 15, Amer. Math. Soc., Providence, RI, 1977.

[9] H. O. Fattorini, Second Order Linear Differential Equations in Banach Spaces, North-Holland, Amsterdam, 1985.

[10] H. R. Henríquez and C. Vásquez, Almost periodic solutions of abstract retarded functional differential equations with unbounded delay, Acta Appl. Math. 57 (1999), 105-132.

[11] - - - Differentiability of the second order abstract Cauchy problem, Semigroup Forum 64 (2002), 472-488.

[12] J. Kisyński, On cosine operator functions and one parameter groups of operators, Studia Math. 49 (1972), 93-105.

[13] C.-M. Marle, Mesures et Probabilités, Hermann, 1974.

[14] S. K. Ntouyas, Global existence results for certain second order delay integrodifferential equations with nonlocal conditions, Dynam. Systems Appl. 7 (1998), 415-425.

[15] S. K. Ntouyas and P. Ch. Tsamatos, Global existence for second order semilinear ordinary and delay integrodifferential equations with nonlocal conditions, Appl. Anal. 67 (1997), 245-257.

[16] C. C. Travis and G. F. Webb, Second order differential equations in Banach space, in: Proc. Internat. Sympos. on Nonlinear Equations in Abstract Spaces, Academic Press, New York, 1987, 331-361.

[17] - - - Compactness, regularity, and uniform continuity properties of strongly continuous cosine families, Houston J. Math. 3 (1977), 555-567.

[18] - - - Cosine families and abstract nonlinear second order differential equations, Acta Math. Acad. Sci. Hungar. 32 (1978), 76-96.

[19] T. J. Xiao and J. Liang, The Cauchy Problem for Higher-Order Abstract Differential Equations, Lecture Notes in Math. 1701, Springer, Berlin, 1998.

[20] S. D. Zaidman, Almost Periodic Functions in Abstract Spaces, Pitman, London, 1985.

Departamento de Matemática

I.C.M.C., Universidade de São Paulo

13560-970 São Carlos, SP, Brasil

E-mail: lalohm@icmc.sc.usp.br
Departamento de Matemática Universidad de Santiago Casilla 307, Correo 2 Santiago, Chile

E-mail: hhenriqu@lauca.usach.cl 